\title{
Inappropriate child feeding practice and primary health care as major correlates of stunting and underweight among infants and young children 6- 23months of age in food insecure households of Amhara and Oromia Regions, Ethiopia. A community based cross sectional study
}

Zelalem Tafese Wondimagegne ( $\nabla$ wudasiez@gmail.com )

Hawassa University College of Agriculture

Fekadu Reta Alemayehu

Hawassa University College of Agriculture

Anchamo Anato Adole

Hawassa University College of Agriculture

Kaleab Baye Yimam

Addis Ababa University College of Natural Sciences

Yifru Berhan

St pauls hospiital millenium medical college

\section{Barbara J Stoecker}

Oklahoma State University Center for Health Sciences

Research article

Keywords: Stunting, zinc supplement, iodized salt, diet diversity, maternal income

Posted Date: August 11th, 2020

DOI: https://doi.org/10.21203/rs.2.12461/v2

License: (c) (i) This work is licensed under a Creative Commons Attribution 4.0 International License.

Read Full License 
The authors have withdrawn this preprint from Research Square 